\title{
Opto-mechanical modelling and experimental approach to the measurement of aerospace materials using shearography and thermal loading
}

\author{
Eduardo C. Krutul ${ }^{\mathrm{a}, \mathrm{b}}$, Roger M. Groves ${ }^{\mathrm{a}}$ \\ ${ }^{a}$ Delft University of Technology, Optical Non-Destructive Testing Laboratory, Faculty of Aerospace \\ Engineering, Kluyverweg 1, 2600 GB Delft, The Netherlands \\ ${ }^{\mathrm{b}}$ Universidade Federal de Santa Catarina, Laboratório de Metrologia e Automatização, Departamento \\ de Engenharia Mecânica, CEP 88040-970, Florianópolis, Brazil
}

\begin{abstract}
This paper is concerned with the modelling of the complete opto-mechanical measurement system, including shearography instrument, loading technique and the response of the object under test and the comparison of the simulation results with experimental results. To show the applicability of this technique, the response of an aluminium flat plate sample under thermal load was analysed. First a finite element model of the plate was generated, using an experimentally measured temperature profile. The strain and displacement values obtained from the finite element model were used to simulate the phase-map in the optical part of the model. The simulated phase maps were then compared qualitatively with experimental phase maps measured using shearography. This approach is suitable to understand the response of components under load and to predict anomalies such as defects, thereby making the analysis of measured phase maps easier and less empirical.
\end{abstract}

Keywords: Shearography, thermal loading, opto-mechanical model.

\section{INTRODUCTION}

The aerospace industry is in the progress of moving from classical materials to lightweight composite and hybrid materials ${ }^{1}$, such as carbon fibre and GLARE. These lightweight materials require advanced measurement techniques that are preferentially fast, non-destructive, non-contact and full-field. Optical methods meet all these requirements and appear as a suitable solution for non-destructive inspection both in the production environment and for maintenance ${ }^{2}$. In particular interferometer and holographic techniques offer a high sensitivity to displacement, allowing components to be assessed non-destructively using small loading levels ${ }^{3}$. Unfortunately many interferometric and holographic techniques are sensitive to disturbances from the measurement environment. Shearography ${ }^{3}$ overcomes this as its common-path interferometer configuration makes it insensitive to small amplitude displacements, such as vibrations.

Meanwhile a detailed knowledge of the measurement environment is necessary to detect defects. Less well known for optical measurements is the relationship between measurement technique, loading method and the material under test. Very often the selection of parameters is made by intuition, experience or trial and error. Evaluation of thermal load can also be made using thermography ${ }^{4}$. In this related technique, the sensor measures the temperature distribution over the sample surface, while when using shearography the derivative of the displacement at the sample surface is measured. Both allow the detection of defects, however by measuring different parameters. Thermal loading is widely applied as a shearography excitation mode for the evaluation of flaws for non-destructive testing (NDT) ${ }^{3}$. Usually commercial heat sources, such as lamps or heat guns are used, making the technique easier to apply compared with other loading techniques, e.g. mechanical or vacuum. Starting to perform tests without the due care for the sample response can lead for a longer overall time to achieve the optimum set up for the shearography system. Also, owing to human interpretation of the phase maps, wrong interpretations of the fringes can be made.

Modeling Aspects in Optical Metrology III, edited by Bernd Bodermann, Harald Bosse, Richard M. Silver, Proc. of SPIE Vol. 8083, 80831C · C 2011 SPIE · CCC code: 0277-786X/11/\$18 · doi: 10.1117/12.889457 
The aim of the research programme is to integrate simulated and measured phase maps in a combined opto-mechanical model, eventually covering a wide range of aerospace materials. In this paper the authors study in detail the measurement of an aluminium sample using shearography and thermal loading. First a finite-element mechanical model is developed to obtain the dynamic mechanical response of the sample under thermal loading. This numerical model is developed using experimentally-derived and reference parameters. An opto-mechanical model of the complete measurement system, including the shearography instrument, is then developed where simulated shearography phase maps will be generated using the data from the finite-element simulation. These phase maps are subsequently compared with experimental results to assess the quality of the model.

\section{SHEAROGRAPHY THEORY}

Shearography is an optical metrology technique, based on the interference of the light. It is closely related to speckle interferometry and holography techniques; however it is less sensitive to environmental disturbances and may be used in an industrial environment ${ }^{3}$. To perform a measurement, the test object is illuminated by coherent light. Light scattered from the object surface is optically mixed in a shearing interferometer and then recorded by a digital camera, see Figure 1.

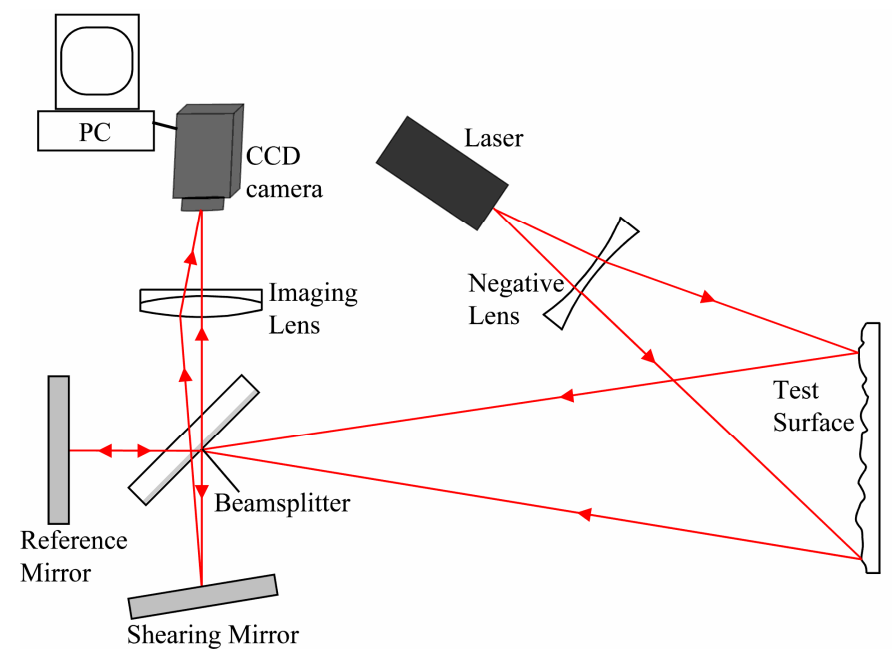

Figure 1 - Modified shearing Michelson interferometer configuration, for experimental shearography.

Shearing mirror should be adjustable to allow tilting in either the $\mathrm{x}$ (horizontal) or y (vertical) directions. Each pixel of the digital camera receives light from two points of the object, so the complete image is an interferogram. This interferogram contains information on the difference in phase of the light between the two points on the sample. To perform a measurement, first is necessary to record a reference interferogram. Then the sample is loaded and a second interferogram is recorded. The interferograms recorded before and after loading are correlated by subtraction to form an image with fringes. These fringes contain information on the sample behaviour under applied load and a concentration of fringes can be interpreted as displacement gradient anomalies which can be a characteristic of the sample boundary conditions or of defects. The phase of the light on each point in the fringe map can be associated with the light path between the laser source $(\mathrm{S})$, two points on the object $\left(\mathrm{P}_{1}\right.$ and $\left.\mathrm{P}_{2}\right)$, the camera sensor $(\mathrm{C})$ and the wavelength $(\lambda)$ of the expanded laser beam as shown on Figure 2. 


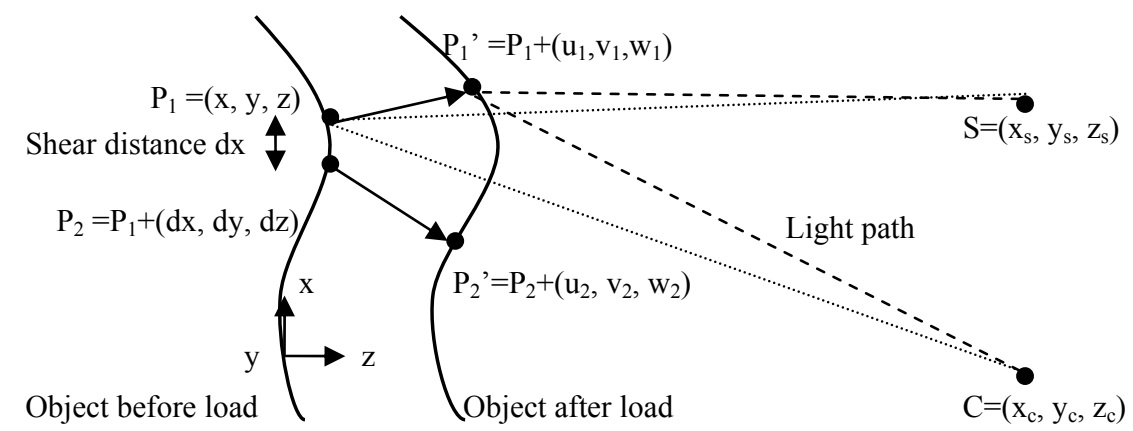

Figure 2 - Diagram of the optical pathlengths in a generalized shearography instrument. $\mathrm{S}$ is the laser source position, $\mathrm{C}$ is the camera position and $\mathrm{P}_{1}$ and $\mathrm{P}_{2}$ are two points on the object surface.

The intensity at each pixel in the interferogram is described by ${ }^{3}$ :

$$
I=2 I_{0}(1+\gamma \cos (\phi))
$$

Where, $\mathrm{I}_{0}=\left(\mathrm{a}_{1}{ }^{2}+\mathrm{a}_{2}{ }^{2}\right) / 2, \gamma=2 \cdot \mathrm{a}_{1} \cdot \mathrm{a}_{2} /\left(\mathrm{a}_{1}{ }^{2}+\mathrm{a}_{2}{ }^{2}\right) \mathrm{a}_{1}$ and $\mathrm{a}_{2}$ are the amplitudes of the light rays from points $\mathrm{P}_{1}$ and $\mathrm{P}_{2}$ on the object surface and $\phi$ is the difference of phase between these two points.

The fringe map can be mathematically described by equation $2^{3}$ where, $\overline{S P_{1} C}$ and $\overline{S P_{2} C}$ are optical path lengths before loading, $\overline{S P_{1}^{\prime} C}$ and $\overline{S P_{2}^{\prime} C}$ are optical path lengths after loading. This phase calculation approach will be used later to calculate simulated phase maps from the finite element model displacement data. The phase at each point in the fringe map is given by:

$$
\Delta \phi=\frac{2 \pi}{\lambda}\left[\left(\overline{S P_{1}^{\prime} C}-\overline{S P_{1} C}\right)-\left(\overline{S P_{2}^{\prime} C}-\overline{S P_{2} C}\right)\right]
$$

Phase-shifting is a technique used to determine the magnitude and direction of the phase change at each camera pixel ${ }^{3}$. It is commonly applied in the interferometer by translating a mirror by pre-defined distances using a piezo-electric phaseshifting device. For example, 3-step phase-shifting, as shown in equation 3, allows the determination of the phase angle, where $I_{1}, I_{2}$ and $I_{3}$ are intensities recorded by the CCD, at different phase shifts.

$$
\phi=\tan ^{-1}\left[\sqrt{3} \frac{I_{3-} I_{1}}{2 I_{2}-I_{1}-I_{3}}\right]
$$

In phase shifting Shearography ${ }^{5}$ the phase is calculated for each loading step on every pixel and phase angles are subtracted, to obtain the map of phase difference.

\section{THERMAL LOADING SIMULATION}

For a better understanding of the experiment, an opto-mechanical model was developed to predict the sample response under thermal load. The opto-mechanical model combines finite element modelling and an optical pathlength calculation. In this section the sample, measurement of the temperature profile and the mechanical and optical simulations will be described. 
A flat plate of isotropic material, aluminium $2024 \mathrm{~T} 3$ with dimensions 300 by 150 by $1 \mathrm{~mm}$, was used to test the approach. This type of aluminium is widely applied for aeronautic structures due to its high-strength properties. The material has the properties shown in table 1 .

Table 1 - Properties of aluminium 2024 T3

\begin{tabular}{l|l|l} 
Elastic Modulus & 72,4 & $\mathbf{G P a}$ \\
\hline Poisson's ratio & 0,33 & -- \\
\hline Density & 2726 & $\mathbf{k g . m}$ \\
\hline Thermal expansion & 23,2 & $\mathbf{1 0}^{-6} \mathbf{K}^{-1}$ \\
\hline Thermal conductivity & 121 & $\mathbf{W} \cdot \mathbf{m}^{-1} \mathbf{K}^{-1}$ \\
\hline Specific Heat & 875 & $\mathbf{J . k g}^{-1} \mathbf{K}^{-1}$
\end{tabular}

The sample holder was chosen to avoid vibration and to be simple to model. The sample was clamped at two edges, which were set as boundary conditions in the simulation. Rotational and translational displacements on the clamped faces were set as null.

\subsection{Temperature profile measurement}

Shearography has a high sensitivity for measuring displacement gradients; consequently a small loading is appropriate. Thermal loading is usually applied using halogen lamps, infrared lamp or heat gun. Halogen lamps and infrared lamp are radiation heat sources, while heat guns use a jet of hot air to transfer heat to the object. The heat gun has two main disadvantages compared with radiation sources: it can generate an undesirable vibration of the object and the introduction of strong air flows interfere with the temperature distribution of the sample. Therefore a $500 \mathrm{~W}$ halogen lamp was used. Six type K thermocouples were glued to known positions on the sample. The thermocouples were placed first on the same side of the sample as the heat source, then on the opposite side of the sample. This procedure is necessary due to the thermal conductivity of the material, which gives a different temperature on the two sides of the sample. Figure 3 shows the temperature change difference at each thermocouple, compared with the initial temperature.
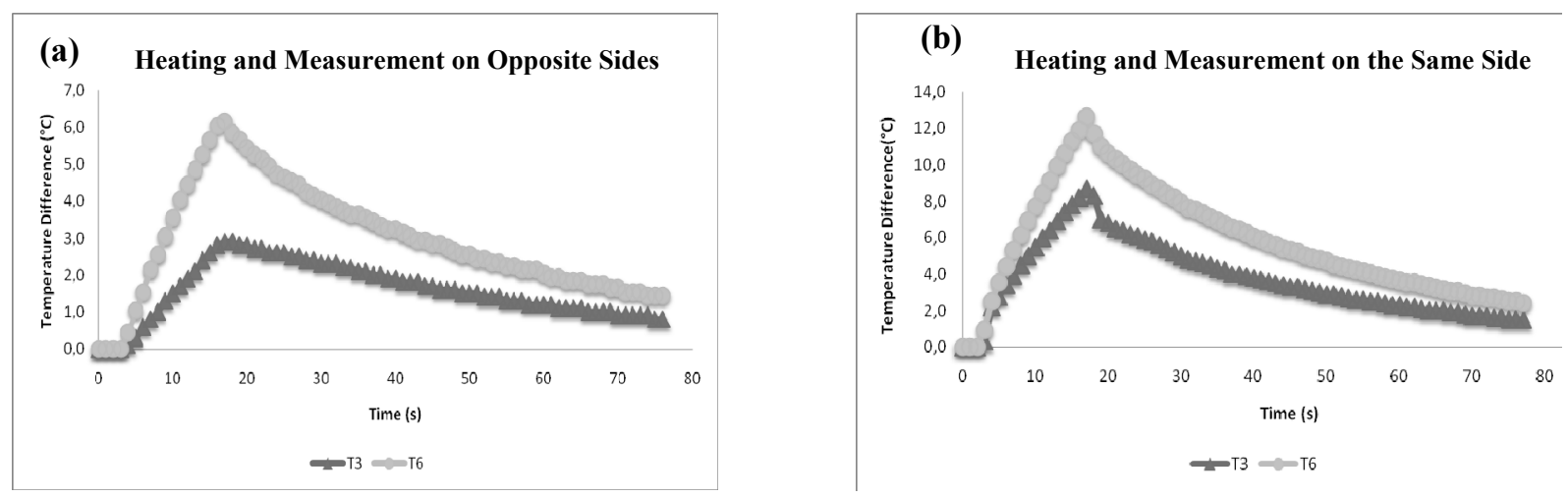

Figure 3 - (a) and (b) show the temperature difference( $\mathrm{Y}$ axis) against time(X axis) on the thermocouple $\mathrm{T} 3$ and $\mathrm{T} 6$, the lowest and highest temperature differences measured.

Graphs (a) and (b) can be separated into four parts, (i) before turning the lamp on, (ii) while the object is heating up, (iii) the moment when the heat source is removed and (iv) the cooling down process. Before turning the lamp on all the thermocouples have the same temperature.

\subsection{Simulation}

To simulate a shearography phase map two states are needed. The before loading state was set as the reference, with the sample at constant temperature. The after loading state was chosen as the time when the sample achieves the largest 
change in temperature over time. This happens just as the cooling phase starts. Using the $\mathrm{x}$ and $\mathrm{y}$ thermocouple positions on the object surface, a $\mathrm{T}(\mathrm{x}, \mathrm{y})$ function was developed to describe the temperature distribution according to position. From the thermocouples readings isotherms with a circular shape are generated. In Cartesian coordinates a 2-D second order function is suitable to describe this circular shape. The equation is shown in equation 5.

$$
T(x, y)=A x^{2}+B y^{2}+C x y+D x+E y+F
$$

The input data for the phase map simulation are the strain and displacement values from the finite element model. This makes the size of the mesh an important issue. A finer mesh can mean a better resolution of the simulated phase maps and a better accuracy on the mechanical model. However the minimum size of the mesh is restricted by the computer capacity of processing. The mesh elements were chosen to allow 3-D displacements. The finite element simulation was performed using Abaqus software (version 6.10) on a PC with an Intel(R) CORETM 2 QUAD CPU Q6600@ 2.4 GHZ and $4 \mathrm{~GB}$ of RAM. Boundary conditions due to the sample holder were set as null displacements.

The shearography simulated phase maps were calculated using the path length method ${ }^{3}$. Data from the finite element model was reorganised to match the shearography measuring area and the position of each node before loading was calculated. The displacement experienced for each node was added to its original position to calculate the position after load. Using the laser wavelength, shear value, camera and laser position is possible to obtain the difference of path length for each node.

\section{EXPERIMENTAL}

The shearography instrument used for the measurements was supplied by Isi-sys $\mathrm{GmbH}$. It has an f-mount $50 \mathrm{~mm}$ lens placed in front of a modified Michelson interferometer. The shearography sensor was attached on a tripod $650 \mathrm{~mm}$ from the sample surface. A diode laser (optical power $100 \mathrm{~mW}$, wavelength $658 \mathrm{~nm}$ ) was placed $500 \mathrm{~mm}$ from the sample and $120 \mathrm{~mm}$ below the shearography sensor. It is desired to have the laser and the camera nearly at the same position and close to the normal to the sample surface ${ }^{3}$. The laser and the sample were fixed to an optical table to prevent undesirable vibration movements. The lamp was positioned approximately $10 \mathrm{~mm}$ behind the sample. The sample heating and image acquisition were synchronised using a LAN interface switch. The interferograms were captured using one PC while a second PC recorded the temperature of the sample. Reference interferograms were first recorded, then after 10 seconds heating of the sample started. During the heating process no images were acquired. After 30 seconds the lamp was turned off and then every 5 seconds an image was captured. The shear applied was $2.5 \mathrm{~mm}$, first in the $\mathrm{X}$, then in the $\mathrm{Y}$ direction. The experimental setup is shown in Figure 4.

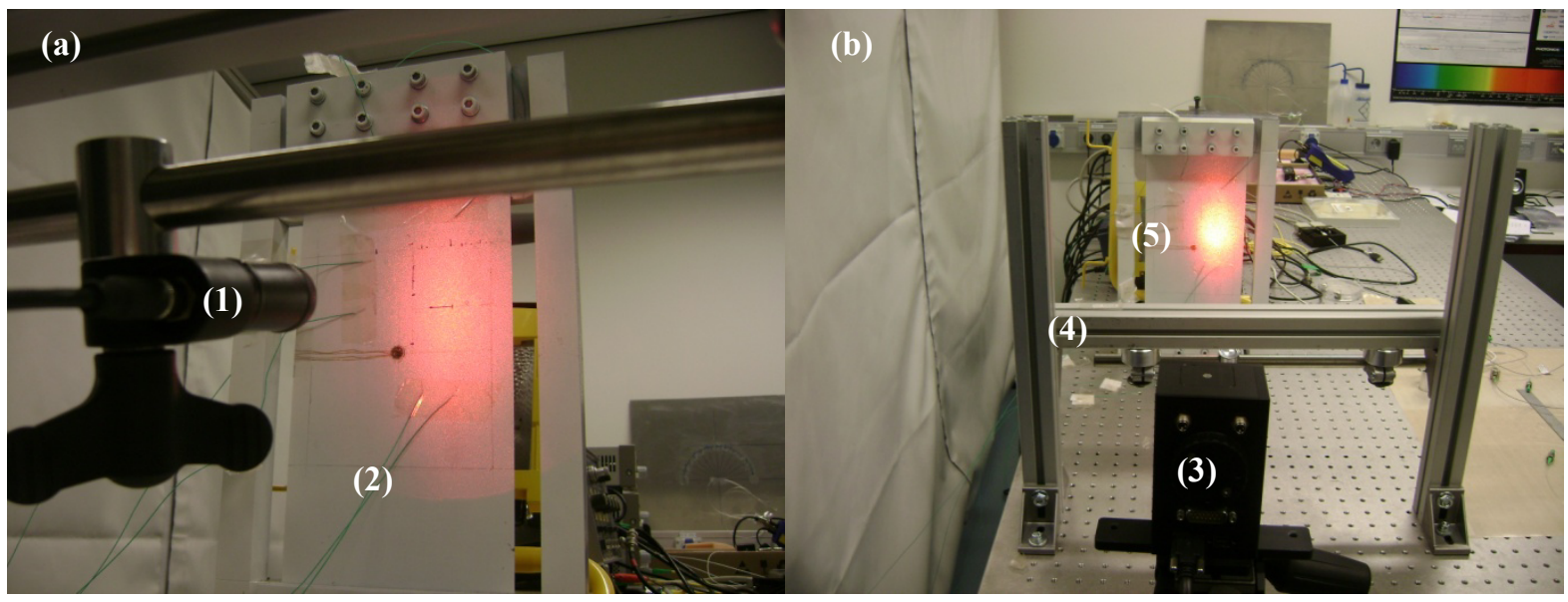

Figure 4 - Picture (a) close up of the sample illumination: (1) the laser source and (2) thermocouples. Picture (b) an overview of the experimental set-up: (3) the Isi-sys sensor, (4) the laser mounting and (5) the sample. 


\section{RESULTS}

\subsection{Different simulated models and measured result}

The opto-mechanical model, described in section 3, was used to simulate phase maps for the loading condition. The field of view covered by the shearography sensor was smaller than the total area of the sample, so a region of interest (ROI) of 85 by $62 \mathrm{~mm}$ was used as the analysis area. To study the effect of the boundary conditions imposed in the model, several simulations were made. In the first simulation the two sides of the sample were clamped. This did not describe the real behaviour of the measured area from experiment. Two further models were developed to analyse the displacement presented on the plate, one without restrictions and another with just one side restricted. The three different models are referenced as $\mathrm{F}$ for the model without restriction; $\mathrm{G}$ with one side restricted and $\mathrm{H}$ with both sides restricted. Figures 5 to 7 present schematic models and associated phase maps. The white squares on the phase maps represent the ROI in Figure 8.

(a)

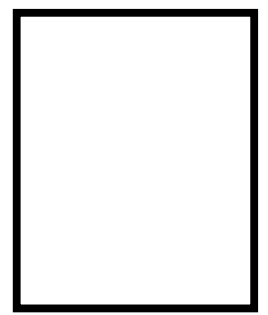

(b)

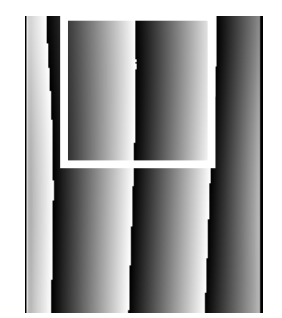

(c)

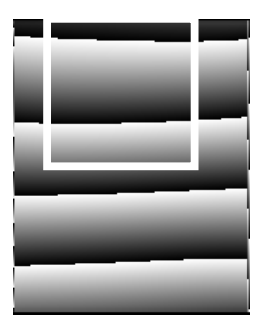

Figure 5 -Schematic of the complete plate (a) without restrictions (model F), (b) and (c) are simulated phase maps with shear on the $\mathrm{x}$ and y directions respectively.

(a)

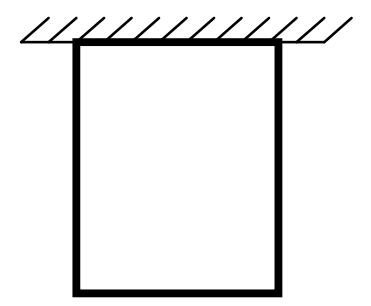

(b)

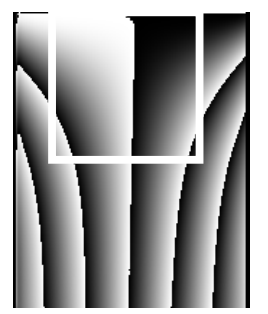

(c)

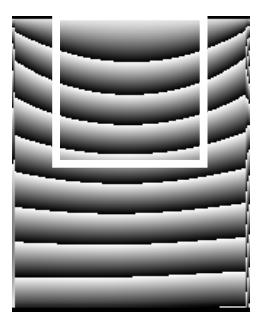

Figure 6 - (a) Schematic mechanical model with just one side restricted (model G), (b) and (c) are simulated phase maps with shear in the $\mathrm{x}$ and $\mathrm{y}$ directions respectively.

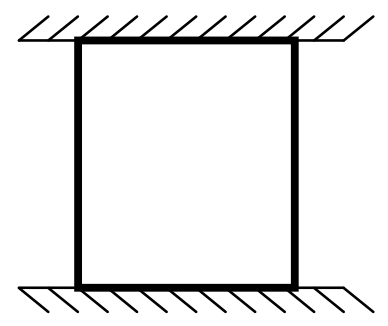

(b)

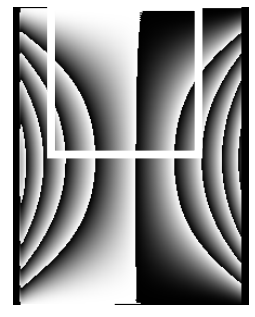

(c)

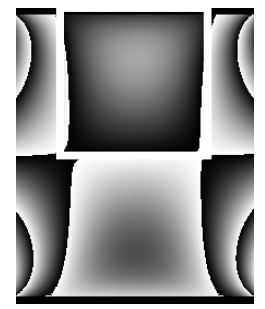

Figure 7 - (a) Schematic mechanical model with both sides restricted (model G), (b) and (c) are simulated phase maps with shear in the $\mathrm{x}$ and $\mathrm{y}$ directions respectively.

The experimental measurements were performed according to the methodology presented in section 4 . The phase maps shown below are the ones that contain the largest phase differences and thus contain the highest displacement gradients. They are obtained from subtracting the interferogram recorded at the highest sample temperature change from that recorded at the reference sample temperature. Figure 8 (a) and (b) presents measured phase maps for $\mathrm{x}$ and $\mathrm{y}$ shear 
directions respectively. In (c) and (d) an additional mechanical load was applied to the sample before following the measurement procedure. This mechanical load was applied using a lever that induces slight buckling on the sample. The phase map obtained from this mechanically pre-loaded sample was different from that predicted by the simulation.

(a)

(c)

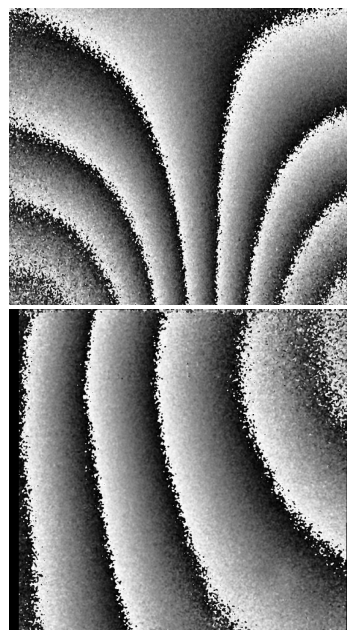

(b)

(d)

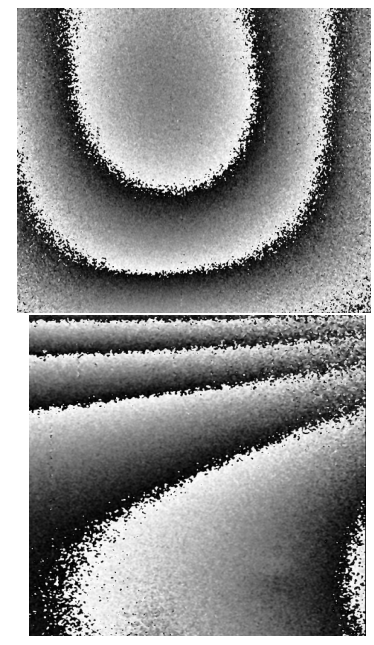

Figure 8 - (a) and (b) measured phase maps using just thermal load on the $\mathrm{X}$ and $\mathrm{Y}$ shear direction respectively. (c) and (d) presents the measured phase maps combining thermal load and pre-load on $\mathrm{X}$ and $\mathrm{Y}$ shear direction respectively.

On the phase map with just the thermal loading is possible to see that the ROI presents a similar behaviour to simulations $\mathrm{G}$ and $\mathrm{H}$. A numerical comparison of the unwrapped phase map with the simulated phase maps was performed by coregistering phase maps and subtracting, for both shear directions. Areas with phase change near to zero show a good match between the model and the experimental phase map. A deviation between the experimental and simulated phase map is shown on both the lower corners.
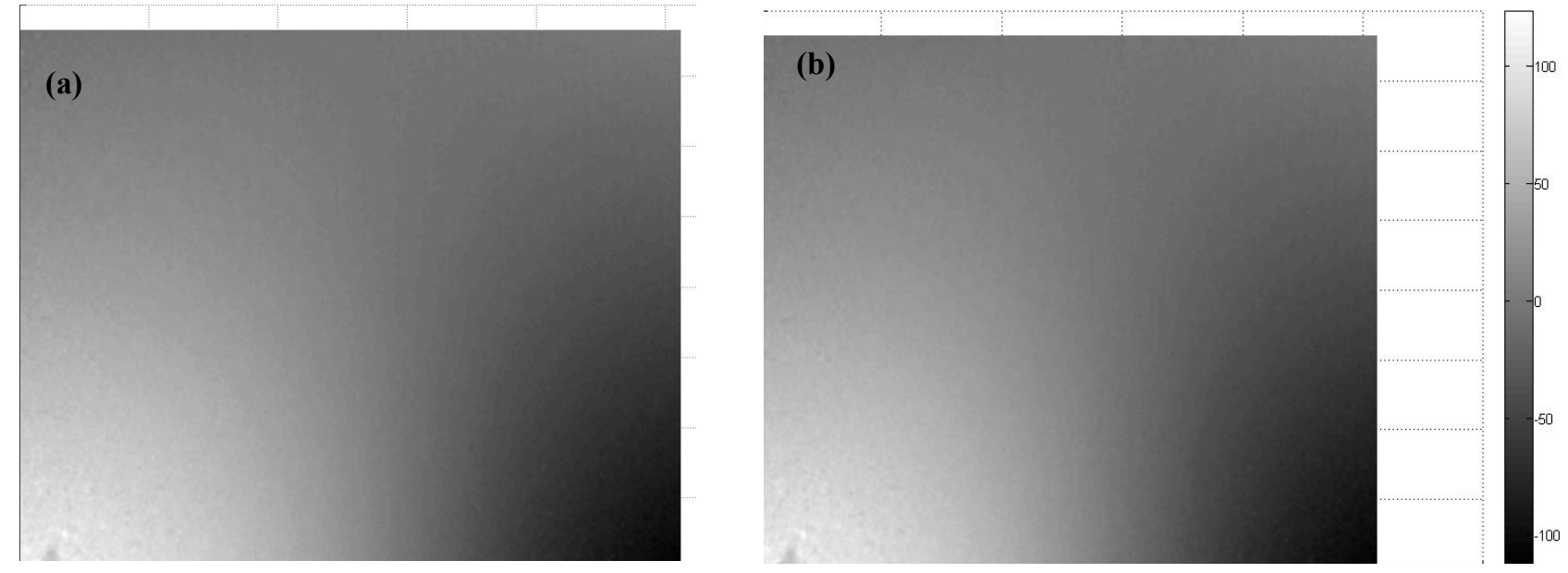

Figure 9 - Subtraction of the simulated unwrapped phase maps with the experimental interferogram for (a) simulation model $\mathrm{G}$ and for (b) simulation model $\mathrm{H}$. 


\section{DISCUSION}

In the experimental phase maps, it is possible to see that the pre-stress has a great influence on the phase maps, which could even lead to an error during an analysis. The shape of the fringes evaluated with the pre-load appears to have a similar shape as the fringes simulated on model F. This could be explained as the mechanical load creating a buckling similar to the thermal load without restrictions. As this has a stronger influence than the thermal load the fringes are dominated by the buckling. Usually this is what happens in shearography for NDT using thermal load, the difference of temperature among the two states is not high, and therefore displacement gradients due to this are not large. A small preexisting load, such as residual stress, can create distortion in the fringes. This could also be an indication of a defect. Using a simulated model before performing measurements it should be possible to have a qualitative evaluation in the presence of pre-stress.

The simulated phase maps are more symmetrical than the experimental ones. This difference can be due to boundary conditions in the model and also because of the experimental set-up. The experimental clamping system could possibly allow the sample to move so the displacement value on the contact area is not null. The camera and sample have slightly different positions experimentally than the set-up for the simulation or even the temperature profile can have differences not fully described by the equation. These parameters are being analysed to improve of the model.

The subtracted unwrapped phase maps show that cases $\mathrm{G}$ and $\mathrm{H}$ could be use to model the problem. Both subtracted phase maps show the same result. The error presented on the lower corners can be because of the difference of symmetry explained in the previous paragraph. A larger area of inspection or a smaller sample is desired for a more accurate evaluation of the model. While the models $\mathrm{G}$ and $\mathrm{H}$ have a similar behaviour only an analysis of the whole object, colleting information on both clamped areas can provide a more accurate comparison. Analysing the images with the shear on the y direction, it is possible to make a qualitative evaluation of the suitability from the model. While the measured wrapped images the fringes appear to develop in the middle of the sample and not from the borders it is possible to say that model $\mathrm{H}$ is the one that best describes the experimental set-up.

In this paper only one heating state was evaluated. This was the interferogram just after heating. Improvements on the model could be done if more states were simulated presenting images on the whole curve of cooling, so the minimum energy necessary to excite the sample could be evaluated making the measurement process much faster. Increasing the shear also increases the measurement sensitivity, however with loss of spatial resolution. The real system appears to be more sensitive to the shear influence than the model. This could be noticed from the number of fringes in the phase maps. This difference does not influence the analysis of this model, because at this stage, the shape of the fringes is more important. However further study is required to evaluate if this difference of fringe concentration, influences a model containing defects.

The temperature profile was evaluated by extrapolating 6 temperature points into a field of temperature, this approach introduces simplifications in the model, consequently adding errors. The applicability of the technique is shown in this paper and appears to be suitable for a non-destructive evaluation. However to use this methodology for a strain measurement, a thermal camera would be required to validate the temperature field used in the simulation.

\section{CONCLUSION}

In this paper the authors demonstrate an opto-mechanical model and experimental approach for the evaluation of an aluminium plate using shearography excited by thermal load. The simulated and experimentally determined phase maps showed a good similarity in form. However improvements of the model are required to study the asymmetry of the experimental phase maps. As a first model, this approach shown appears suitable for future use in industrial environments. The simulated experimental approach gives more arguments for evaluating shearography fringes than just performing tests. Further investigations involving samples manufactured using anisotropic materials, samples with defects and more complex shapes are the next focus of research. 


\section{ACKNOWLEDGEMENTS}

The authors gratefully acknowledge Dr Peter Mäckel, Isi-sys GmbH for the loan of the shearography instrument and TU Delft for partial funding of this project. They would also like to acknowledge the support provided by the colleagues of Isi-sys GmbH, They would to thank Dênis Takeo Goto and the research team of the Laboratory of Metrology and Automation (UFSC) for their support during the research.

\section{REFERENCES}

[1] Noor, A., K., Vennei, S., L., Paul, D., B. and Hopkins, M., A., "Structure technology for future aerospace system", Computer and Structures, 74, 507-19 (2000).

[2] Wilhem, T., Hinnen, M., Schmdit, W., Montnacher, J. and Verl, A., "Non-destructive testing of adhesive joints", Adhäsion Kleben \& Dichten, 00010 (2010).

[3] Steinchen, W. and Yang, L., [Digital Shearography], SPIE Press, Bellingham, Washington (2003).

[4] Bøving, K. G., ed. [NDE Handbook - Non-destructive examination methods for condition monitoring], Woodhead Publishing Limited, Cambridge, UK (2009).

[5] Robinson, D. W. and Reid, G. T., eds. [Interferogram Analysis - Digital Fringe Pattern Measurement Techniques], Institute of Physics Publishing, Bristol, UK (1993).

[6] Davis, C. K., "Shearography and thermographic non-destructive evaluation of the space shuttle structure and thermal protection (TPS) Systems“, Proc SPIE 2945, 36-47 (1996).

[7] Ettemeyer A., "Combination of 3-D deformation and shape measurement by electronic speckle pattern interferometry for quantitative strain stress- analysis", Opt. Eng., 39, 212-215 (2000).

[8] Goto, D.T., Groves, R.M., "Error analysis of 3D shearography using finite-element modelling", Proc. SPIE 7718, 771816, 1-11 (2010).

[9] Steinchen,W. Yang.L., “TV-shearography for measuring 3-D strains”, Strain, 32, 49-58 (1996).

[10] Francis, D. Tatam, R.P., Groves, R.M., "Shearography technology and applications: a review", Meas. Sci. Technol., 21, 102001, 29pp (2010).

[11] Incropera, F.P, Dewit, D.P, [Fundamentals of Heat and Mass Transfer] John Wiley \& Sons, Fifth Edition (2002).

[12] Goto, D.T., Groves, R.M., "A combined experimental with simulation approach to calibrated 3D strain measurement using shearography”, Proc. SPIE 7387, 77871J (2010).

[13] Georges, M., Thizy, C., Vandenrijt, J-F., Alexeenko, I., Pedrini, G., Osten, W., Aldave, I.J., Lopez, I., Saez de Ocariz, I. Vollheim, B., Dammass, G. and Krausz, M., " Fantom project: electronic speckle pattern interferometry at thermal infrared wavelenghts, a new technique for combining temperature and displacement measurements", EASN Workshop, Paris, France, (2010).

[14] Isi-sys GmbH, [User Manual and Service Instructions SE3 Shearography / ESPI], (2010). 